\title{
All-Cause and Cardiovascular Mortality Among Insulin-Naïve People With Type 2 Diabetes Treated With Insulin Detemir or Glargine: A Cohort Study in the UK
}

Lise Lotte Nystrup Husemoen • Lina S. Mørch • Per K. Christensen •

Niels V. Hartvig · Michael D. Feher

Received: December 10, 2020 / Accepted: March 4, 2021 / Published online: March 15, 2021

(C) The Author(s) 2021

\section{ABSTRACT}

Introduction: Uncontrolled type 2 diabetes (T2D) is associated with an increased risk of micro- and macrovascular complications and mortality. The impact of basal insulins on the risks of mortality and cardiovascular mortality in people with T2D has not been thoroughly investigated in real-world settings. The aim of the present real-word study was to investigate differences in mortality among insulin-naïve people with T2D who initiated insulin detemir (detemir) and insulin glargine (glargine).

Methods: We assessed all-cause and cardiovascular mortality in people with T2D, aged $\geq 40$ years and insulin-naïve at treatment initiation. People were identified from the United Kingdom Clinical Practice Research Datalink GOLD national database (2004-2019).

Supplementary Information The online version contains supplementary material available at https:// doi.org/10.1007/s13300-021-01048-4.

L. L. N. Husemoen $(\varangle)$ · L. S. Mørch .

P. K. Christensen · N. V. Hartvig

Novo Nordisk A/S, Søborg, Denmark

e-mail: lshu@novonordisk.com

M. D. Feher

Chelsea and Westminster Hospital, London, UK

M. D. Feher

Nuffield Department of Primary Care Health

Sciences, University of Oxford, Oxford, UK
Database information included prescribed medications, demographic and clinical variables and mortality. Cause of death was obtained from the Office for National Statistics (ONS). For mortality, 24 clinically relevant confounders were considered and adjusted for using Cox regression analyses.

Results: The total cohort included 12,847 people with T2D, including 3031 who commenced detemir and 9816 who commenced glargine. Median age was 66.8 years and median diabetes duration was 7.6 years. From the total cohort, 3231 deaths occurred during follow-up and 6897 people were eligible for linkage to the ONS for cardiovascular mortality data (528 cardiovascular deaths). The adjusted hazard ratio (HR) (95\% confidence interval [CI]) was $0.86(0.79$; $0.95)$ for all-cause mortality and 0.83 (0.67; 1.03) for cardiovascular mortality, in favour of detemir versus glargine. These associations were more pronounced among people with obesity (body mass index $\left.\geq 30 \mathrm{~kg} / \mathrm{m}^{2}\right)$, with HRs $(95 \%$ CI) of $0.79(0.69 ; 0.91)$ and $0.69(0.50 ; 0.96)$ for all-cause and cardiovascular mortality, respectively.

Conclusion: In this real-world observational study, there was an association between allcause mortality and basal insulin choice in insulin-naïve people with T2D; the mortality risk was lower with detemir versus glargine after adjustment for potential confounders. 
Keywords: Basal insulin; Cardiovascular mortality; Cohort study; Insulin detemir; Insulin glargine; Insulin therapy; Long-acting insulin analogues; Mortality; Type 2 diabetes

\section{Key Summary Points}

\section{Why carry out this study?}

The impact of basal insulin choice on the risks of all-cause and cardiovascular mortality has not been thoroughly investigated in a real-world setting for people with type 2 diabetes (T2D) from the UK.

A previous real-world study in Finland observed a lower mortality risk associated with insulin detemir (detemir) than with insulin glargine (glargine), which they hypothesised might be explained by a difference in cardiovascular mortality.

\section{What was learned from the study?}

In the present real-world study in the UK, there was a lower risk of all-cause mortality in insulin-naïve people with T2D who initiated detemir than in those who initiated glargine, after adjustment for potential confounding factors.

There was also a non-significant trend towards a lower risk of cardiovascular mortality associated with detemir versus glargine, again after adjustment for potential confounders.

Of potential interest, the lower risks of all-cause and cardiovascular mortality associated with detemir in comparison with glargine were most pronounced in a subset of people with body mass index $\geq 30 \mathrm{~kg} / \mathrm{m}^{2}$.

\section{DIGITAL FEATURES}

This article is published with digital features, including a summary slide, to facilitate understanding of the article. To view digital features for this article go to https://doi.org/10.6084/ m9.figshare.14153456.

\section{INTRODUCTION}

Poor glucose control in diabetes may result in an increased risk of complications, including microvascular and macrovascular disease [1], with an increase in early mortality [2]. In advanced type 2 diabetes (T2D), where non-insulin therapies are not able to maintain glycaemic control, basal insulin therapy is frequently added to achieve optimal glycaemic control with the aim to reduce the risk of both acute and chronic complications [3].

Both insulin detemir (detemir) and insulin glargine (glargine) are long-acting insulin analogues, administered subcutaneously via injection, with a duration of action of up to, or over, $24 \mathrm{~h}$ depending on the product and dose $[4,5]$. Both insulins are a pharmacological advance compared to human insulins, but differ in their pharmacokinetic/pharmacodynamic profiles, resulting in differing propensity for causing weight gain, hypoglycaemia and periods of hyperglycaemia or variability in blood glucose levels $[6,7]$. As these factors may all contribute to cardiovascular risk, it is possible that different basal insulins may affect prognosis differently. However, there is limited knowledge of the differences between the available basal insulins regarding their impact on the risk of cardiovascular disease (CVD) and early mortality.

In randomised controlled trials (RCTs) $[8,9]$ and short-term observational studies $[10,11]$, detemir has shown advantages in terms of less weight gain compared with glargine. To assess the effect of a clinical intervention on mortality, many participants would need to be followed for a long time in RCTs, with surrogate or composite endpoints often utilised instead $[12,13]$. Furthermore, the more strict inclusion and exclusion criteria for trial enrolment (in comparison with real-world studies) mean that 
the results of RCTs tend to be less applicable to the general populations seen in clinical practice [14].

A long-term observational study conducted in Finland observed that detemir was associated with a significantly lower all-cause (29\% lower) and cardiovascular (36\% lower) mortality risk than glargine [11]. To the authors' knowledge, this has been the only real-world study to date to compare the all-cause and cause-specific mortality risk in people with T2D treated with detemir in comparison with glargine. Moreover, these findings need to be assessed in other patient populations and with use of other data sources with different sources of potential bias to strengthen the evidence base for these observations and test their generalisability across different settings and patient populations [15].

Analyses of real-world data from large databases, such as the Clinical Practice Research Datalink (CPRD), can be useful to assess response to, and outcomes associated with, T2D treatments, and hence can augment current clinical trial research. Therefore, the aim of this register-based cohort study in the UK was to estimate the differences in all-cause and cardiovascular mortality rates between first-time users of the basal insulins detemir and glargine in a real-world population with T2D by utilising the CPRD GOLD database.

\section{METHODS}

The study protocol was approved by the Independent Scientific Advisory Committee (ISAC) (Medicine and Healthcare Products Regulatory Agency): approved study protocol number 19_058A.

\section{Study Cohort}

The study cohort was identified in the CPRD GOLD national database: a large, primary care database that includes data from people registered at general practices across the UK (England, Wales, Scotland and Northern Ireland). The database contains information on people's demographics, medical diagnoses and symptoms, prescribing information and lifestyle factors, including alcohol consumption and smoking. Individuals in the database can be linked to secondary care and other health datasets, enabling a wider view of patient care [16].

Eligible people in the database were those with T2D, who were insulin-naïve, aged $\geq 40$ years and who initiated treatment with either detemir or glargine between 24 June 2004 and 10 May 2019, in accordance with local guidelines (Fig. 1). People with T2D were defined as those with a record of a read code for T2D (Appendix 1) and without a record of a read code for T1D (at any time) in the database (Appendix 2). People who initiated detemir (Appendix 3) and glargine (Appendix 4) were defined as those with a record of a prescription for the appropriate insulin at or after 24 June 2004 (the date on which both drugs were available in the database and thus were

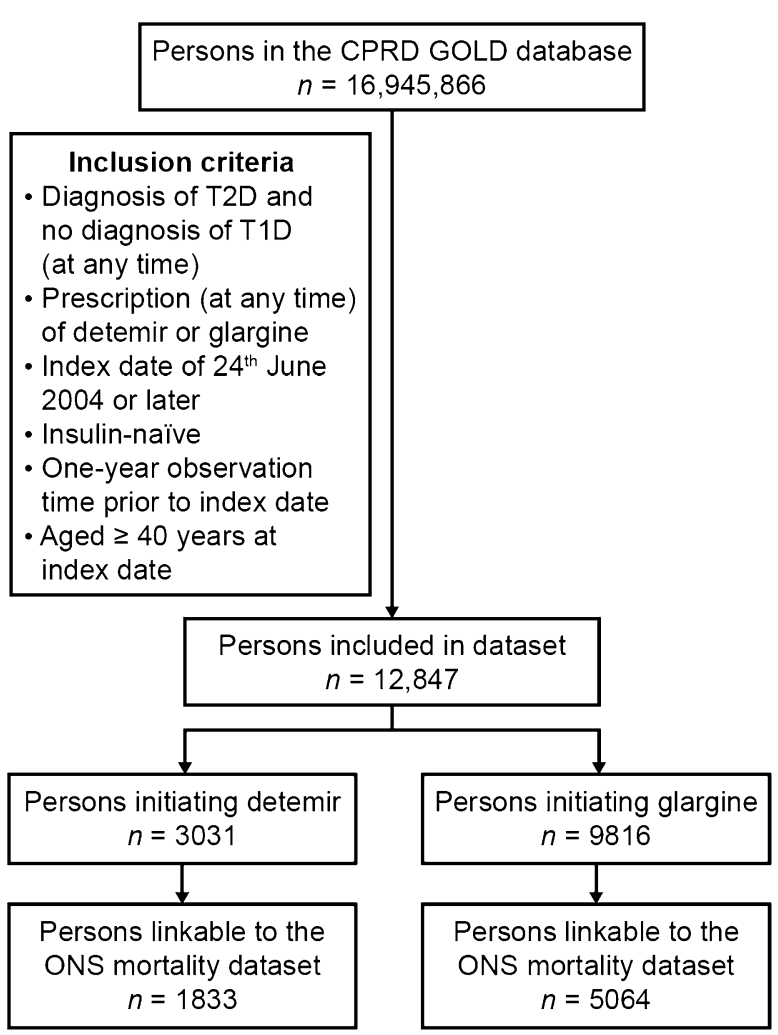

Fig. 1 Study cohort disposition. CPRD Clinical Practice Research Datalink, detemir insulin detemir, glargine insulin glargine, ONS Office for National Statistics, T1D type 1 diabetes, $T 2 D$ type 2 diabetes 
available for prescription in the UK). Eligible people who initiated glargine could receive either the 100 units/ml (U100) or 300 units/ml (U300) formulation. Insulin-naïve people were defined as those without a previous record of a prescription for any insulin (Appendix 5) prior to their prescription for detemir or glargine. Eligible people were those who, at least 1 year prior to initiating detemir or glargine, had first registered with the practice and had acceptable research-quality data (as defined by the CPRD [17]) and the practice was of acceptable research quality (as defined by the CPRD [17]).

\section{Outcomes}

All-cause mortality was defined as mortality from any cause and was identified in the CPRD GOLD database through a registration of mortality date. Cardiovascular mortality was defined as mortality from CVD and was identified through linkage between the CPRD GOLD database and the Office for National Statistics (ONS) death registration data, which includes information on the official date and cause(s) of death [16]. Specifically, mortality from CVD was identified as a record with the 10th revision of the International Statistical Classification of Diseases and Related Health Problems (ICD-10) codes I00-I99 specified as the underlying cause of death (Supplementary Table S1). Linkage was not available for the total study cohort but was available for practices based in England and Wales that had consented to participate in the linkage scheme.

\section{Follow-up Time}

The index date was defined as the first prescription date for detemir or glargine. End of follow-up was defined as the earliest date the patient transferred out of the practice or the date of the last collection for the practice in the CPRD GOLD database, or the end of ONS coverage (14 January 2019). Total follow-up time was calculated as the time (in years) from the index date until death or end of follow-up, whichever came first.

\section{Baseline Characteristics}

Socioeconomic characteristics were obtained by linking the CPRD GOLD database to small arealevel databases (patient and practice level of index of multiple deprivation [IMD2015]). Data for all other baseline characteristics were obtained from the CPRD GOLD database. These included the following characteristics: age, sex (male, female), calendar year of index date (2004-2019), diabetes duration, smoking status (current, former, never) and glycated haemoglobin (HbA1c). Body mass index (BMI) was calculated from weight and height (at age $\geq 18$ years). Dyslipidaemia was defined as total cholesterol $>6.2 \mathrm{mmol} / \mathrm{l}$ or low-density lipoprotein cholesterol $>6.2 \mathrm{mmol} / \mathrm{l}$ or highdensity (HDL) cholesterol $\leq 1 \mathrm{mmol} / \mathrm{l}$ for men or HDL cholesterol $\leq 1.2 \mathrm{mmol} / \mathrm{l}$ for women or triglyceride $\geq 1.7 \mathrm{mmol} / \mathrm{l}$. Hypertension was defined as systolic blood pressure $\geq 140 \mathrm{mmHg}$ or diastolic blood pressure $\geq 90 \mathrm{mmHg}$. Kidney function was ascertained by estimated glomerular filtration rate (eGFR) calculated according to the Cockcroft-Gault formula: eGFR in $\mathrm{ml} / \mathrm{min} / 1.73 \mathrm{~m}^{2}=(140-$ age in years) $\times$ weight in $\mathrm{kg} \times 1.23$ for men, 1.03 for women)/serum creatinine in $\mu \mathrm{mol} / \mathrm{l}$. The latest values prior to the index date were used for weight, height, HbA1c, dyslipidaemia and systolic and diastolic blood pressure. History of CVD, prior hypoglycaemia, nephropathy, retinopathy, neuropathy, chronic kidney disease and cancer were defined as a record of selected read codes prior to the index date (Appendix 6). Bolus insulin, non-insulin glucose-lowering medication and CVD-preventive medication were defined as records of selected product codes within 1 year prior to the index date (Appendix 7).

\section{Statistical Analyses}

Data import and pre-processing were performed in SAS Enterprise Guide 7.15. Statistical analyses were performed using $\mathrm{R}$ version 3.6.0 and the "survival" package version 2.44-1.1. Total basal insulin exposure during follow-up was further examined by plotting the years of cumulative 
exposure against the time since the index date. Crude mortality rates were calculated as the number of deaths divided by the total follow-up time and reported as rates per 100 person-years. Furthermore, cumulative event curves showed the probability of mortality versus the time since first prescription.

\section{Main Analyses}

Cox proportional hazards models were used to calculate crude and adjusted hazards ratios (HRs) with 95\% confidence intervals (CIs) for all-cause and cardiovascular mortality in people treated with detemir relative to glargine. The main analyses were intention-to-treat-like analyses where people were allocated to the treatment group of their first prescription during the entire follow-up period. Age was used as the underlying timescale in the Cox proportional hazards models. Both the crude and adjusted models included the calendar year of the index date (2004-2019) as a categorical variable. Furthermore, the following pre-specified covariates that were expected to be the most likely potential confounders (Table 1) were included in the adjusted models: sex (male, female), diabetes duration (0-1, 1-2, 2-5, $5-10,>10$ years), BMI (categorical, based on World Health Organization classes), history of CVD (yes, no), CVD medication at baseline (antihypertensive [yes, no]; lipid lowering [yes, no]; other cardiovascular [yes, no]), sodiumglucose cotransporter-2 inhibitor and/or glucagon-like peptide- 1 receptor agonist (time-varying: yes, no); other non-insulin glucoselowering medications (time-varying: yes, no) and bolus insulin (time-varying: yes, no; data not summarised in Table 1).

In addition to the pre-specified covariates, the fully adjusted models considered the following potential baseline covariates: socioeconomic status (level 1, 2, 3, 4 or 5), smoking status (yes, no), HbA1c (continuous), prior hypoglycaemia (yes, no), nephropathy (yes, no), retinopathy (yes, no), neuropathy (yes, no), chronic kidney disease (yes, no), dyslipidaemia (yes, no), hypertension (yes, no), eGFR (continuous) and history of cancer (yes, no). Due to missing data for some of the additional variables considered, potential covariates were selected using a forward one-step procedure and the complete case population (people without missing data for any of the variables). A covariate was selected for inclusion in the fully adjusted model if the HR of detemir versus glargine changed by $>10 \%$ on the natural logscale. The fully adjusted model was then refitted to the total cohort.

The models were verified by plots of Martingale residuals. Since the results from the pre-specified adjusted and fully adjusted models were very similar, only the results from the crude and fully adjusted models are presented in the results section.

\section{Sub-analyses}

The main analyses were repeated for the following subsets of people with cardiovascular risk factors at the index date: those with obesity $\left(\mathrm{BMI} \geq 30 \mathrm{~kg} / \mathrm{m}^{2}\right)$; those with duration of diabetes $\geq 5$ years; those treated with CVD medication and/or with prior CVD; those with HbA1c above the median level for the total cohort.

\section{Sensitivity Analyses}

To investigate the sensitivity of assumptions in the statistical models, the main analyses were repeated for four additional 'sensitivity' analyses: (1) people were censored at the time that their prescription data indicated a switch to a different basal insulin from their first prescription during follow-up; (2) all people were censored after 8 years of follow-up; (3) only those with an index date post-2006 were included in the analysis; (4) a time-varying exposure model was used, which allowed for interruptions to basal insulin use. In sensitivity analysis 4 , a person was assigned to a period of their 'current prescription' if at least one prescription was issued within the previous year; otherwise, the period was defined as 'no current prescription', and people were censored if they switched to a different basal insulin from their first prescription. The HRs (detemir/glargine) in sensitivity analysis 4 were estimated for periods with the 'current prescription'. 
Table 1 Baseline characteristics of the study cohort in relation to the model covariates

\begin{tabular}{|c|c|c|}
\hline Characteristics & Detemir & Glargine \\
\hline$n$ & 3031 & 9816 \\
\hline Follow-up time & $3.86(1.74-6.72)$ & $3.59(1.54-6.72)$ \\
\hline Age at index date & $65.1(56.8-74.5)$ & $67.4(57.7-76.5)$ \\
\hline \multicolumn{3}{|l|}{ Covariates included in simple model } \\
\hline \multicolumn{3}{|l|}{ Year of index date $[n(\%)]$} \\
\hline 2004 & $35(1.2)$ & $380(3.9)$ \\
\hline 2005 & $167(5.5)$ & $821(8.4)$ \\
\hline 2006 & $183(6.0)$ & $815(8.3)$ \\
\hline 2007 & $248(8.2)$ & $816(8.3)$ \\
\hline 2008 & $284(9.4)$ & $921(9.4)$ \\
\hline 2009 & $363(12.0)$ & $844(8.6)$ \\
\hline 2010 & $410(13.5)$ & $775(7.9)$ \\
\hline 2011 & $281(9.3)$ & $772(7.9)$ \\
\hline 2012 & $286(9.4)$ & $701(7.1)$ \\
\hline 2013 & $236(7.8)$ & $691(7.0)$ \\
\hline 2014 & $185(6.1)$ & $621(6.3)$ \\
\hline 2015 & $155(5.1)$ & $580(5.9)$ \\
\hline 2016 & $101(3.3)$ & $487(5.0)$ \\
\hline$\geq 2017$ & $97(3.2)$ & $592(6.0)$ \\
\hline \multicolumn{3}{|l|}{ Covariates included in pre-specified model } \\
\hline Diabetes duration $^{\mathrm{a}}$ (years) & $7.7(4.3-11.6)$ & $7.6(4.1-11.8)$ \\
\hline Sex: female $(n)$ & $1351(44.6 \%)$ & $4387(44.7 \%)$ \\
\hline Prescriptions of GLP-1 RA or SGLT2 $\mathrm{i}^{\mathrm{b}}(\%)$ & 16.4 & 11.8 \\
\hline Prescriptions of other glucose-lowering medication ${ }^{\mathrm{b}}(\%)$ & 96.6 & 95.9 \\
\hline Prescriptions of antihypertensive medication (\%) & 48.9 & 41.5 \\
\hline Prescriptions of lipid-lowering medication (\%) & 48.8 & 40.7 \\
\hline Prescriptions of other CVD medication (\%) & 33.9 & 29.7 \\
\hline $\mathrm{BMI}^{\mathrm{c}}\left(\mathrm{kg} / \mathrm{m}^{2}\right)$ & $30.7(26.8-35.4)$ & $30.0(26.2-34.3)$ \\
\hline CVD history (\%) & 27.0 & 30.5 \\
\hline \multicolumn{3}{|l|}{ Additional covariates, included if relevant } \\
\hline Hypertension (\%) & 36.7 & 38.3 \\
\hline Current smoking (\%) & 16.1 & 16.1 \\
\hline CKD (\%) & 26.0 & 24.4 \\
\hline
\end{tabular}


Table 1 continued

\begin{tabular}{lll}
\hline Characteristics & Detemir & Glargine \\
\hline Dyslipidaemia (\%) & 79.5 & 79.3 \\
HbAlc (\%) & $9.58(8.46-11.00)$ & $9.58(8.40-11.10)$ \\
eGFR (ml/min/1.73 m²) & $80.8(53.7-107.2)$ & $75.7(50.0-102.5)$ \\
Cancer history (\%) & 8.1 & 9.3 \\
Retinopathy (\%) & 26.9 & 27.7 \\
Nephropathy (\%) & 1.0 & 1.5 \\
Neuropathy (\%) & 6.3 & 6.5 \\
History of hypoglycaemia (\%) & 4.5 & 3.6 \\
Lowest socioeconomic status $^{\mathrm{d}}(\%)$ & 18.6 & 22.0 \\
\hline
\end{tabular}

Continuous variables are listed as median (interquartile range)

$B M I$ body mass index, $C K D$ chronic kidney disease, $C V D$ cardiovascular disease, detemir insulin detemir, $e G F R$ estimated glomerular filtration rate, glargine insulin glargine, GLP-1 RA glucagon-like peptide-1 receptor agonist, HbAlc glycated haemoglobin, IMD2015 index of multiple deprivation 2015; $n$ number of people, $S G L T 2 i$ sodium-glucose cotransporter-2 inhibitor, $W H O$ World Health Organization

a Included as a factor in the model, grouped in approximate quintiles: [0;1], [1;2], [2; 5], [5; 10], > 10 years

${ }^{b}$ Included as time-dependent covariate. Baseline prescription data are presented in Supplementary Table S2

c Included as a factor with levels underweight, normal weight, obesity class I, II and III as per WHO definitions

${ }^{d}$ IMD2015 5 socioeconomic status included as a factor with five levels

\section{RESULTS}

\section{Study Cohort}

The total cohort comprised 12,847 people (detemir, $n=3031$, and glargine, $n=9816$ ) (Fig. 1 ). Of the total cohort, $53.7 \%$ (6897 people) were eligible for linkage to the ONS for cardiovascular mortality data (detemir, $n=1833$, and glargine, $n=5064)$. In the total cohort, the median (interquartile range [IQR]) age was 66.94 (57.50-76.09) years (Table 1). Median (IQR) diabetes duration was similar for people in both the detemir and glargine groups, 7.66 (4.28-11.56) vs. $7.61 \quad(4.08-11.81)$ years, respectively. Median (IQR) follow-up time was 3.86 (1.74-6.72) vs. 3.59 (1.54-6.72) years for the detemir and glargine groups, respectively. After 5 years, the median (IQR) exposed time was $4.64(1.93-5.00)$ and 5.00 (2.93-5.00) years for the detemir and glargine groups, respectively (Supplementary Fig. S1). Fewer than 15\% of people switched to another basal insulin during follow-up (14.4\% and 9.7\% with detemir and glargine, respectively).

\section{All-Cause and Cardiovascular Mortality}

The cumulative event curves for all-cause mortality are shown in Fig. 2a. Among the 12,847 people included in the total cohort, there were 3231 deaths: 635 with detemir and 2596 with glargine. The cumulative event curves for cardiovascular mortality are shown in Fig. $2 \mathrm{~b}$. Of the 6897 people linked to the ONS, 528 died of cardiovascular causes, 117 of whom had been prescribed detemir and 411 glargine.

\section{Main Analyses}

For all-cause mortality, the crude and fully adjusted HRs (95\% CI) were 0.85 (0.77; 0.92) and $0.86(0.79 ; 0.95)$, respectively, significantly in favour of detemir (Fig. 3a). For cardiovascular 
(a)

Strata: + Glargine + Detemir

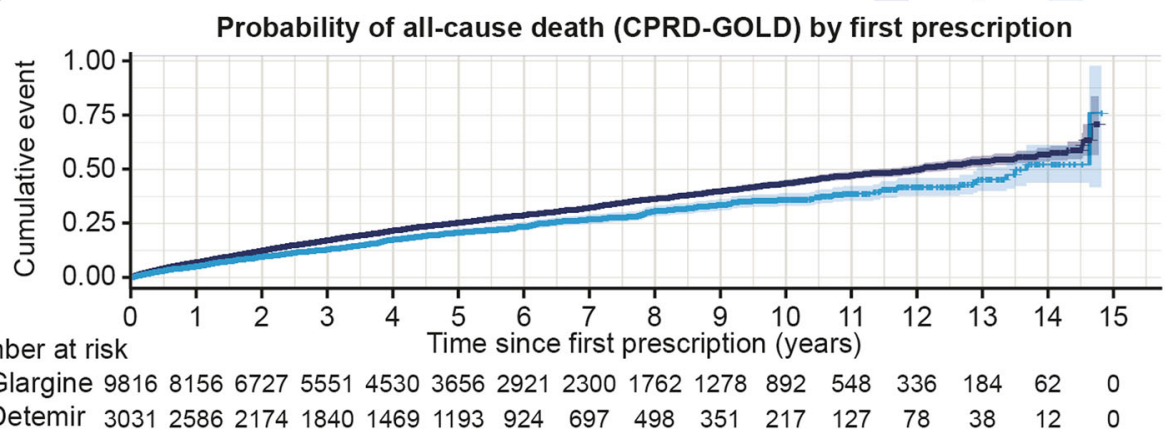

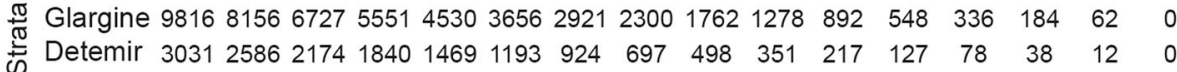

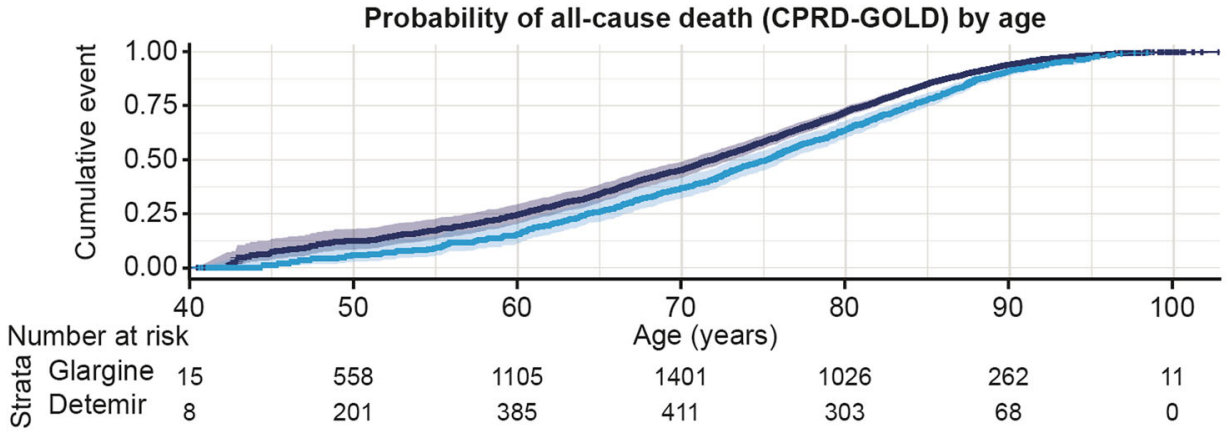

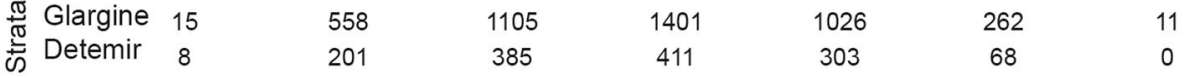

(b)

\begin{tabular}{|l|l|l|}
\hline Total number of all-cause deaths 3231 & Detemir 635 & Glargine 2596 \\
\hline
\end{tabular}

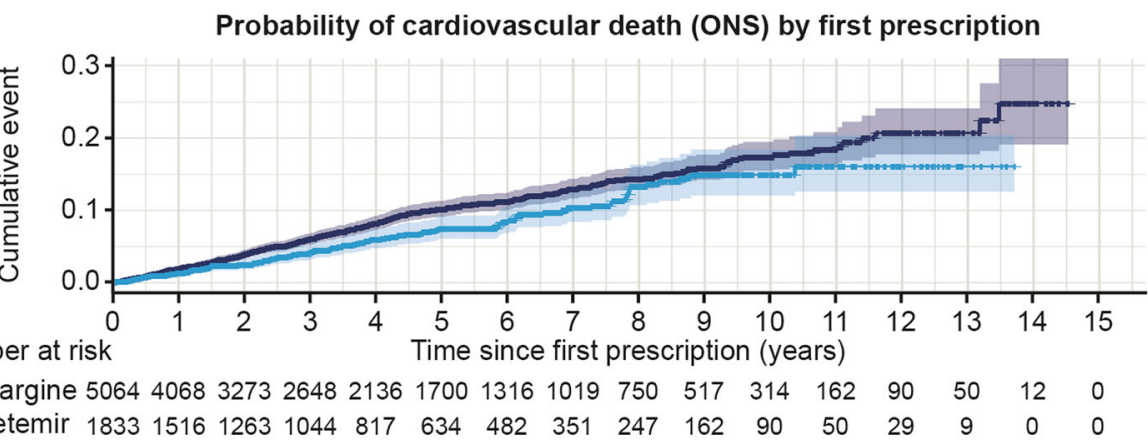

Number at risk

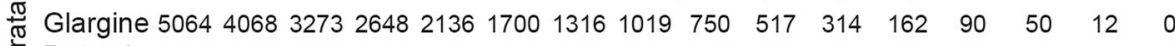

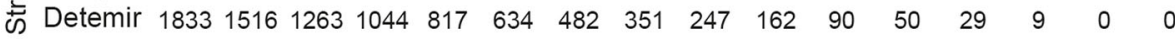

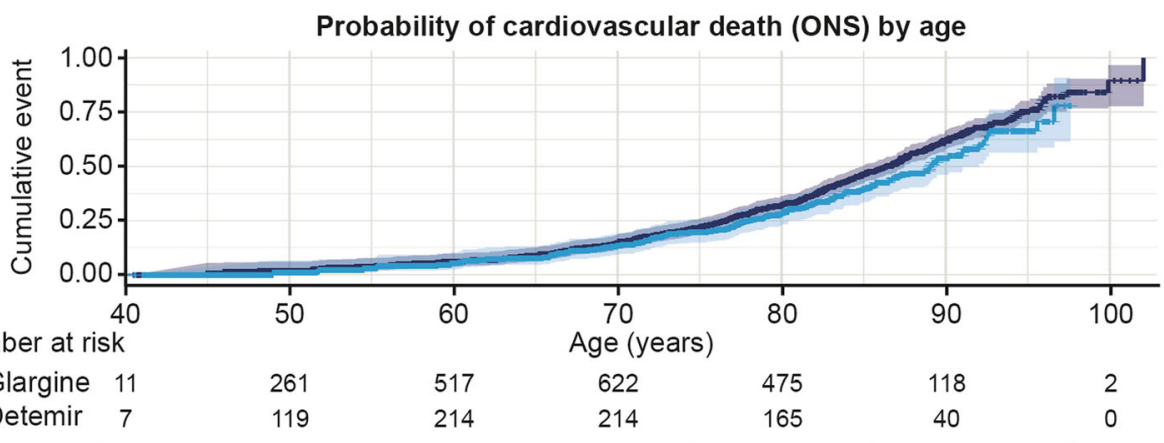

\begin{tabular}{|l|l|l|}
\hline Total number of cardiovascular deaths 528 & Detemir 117 & Glargine 411 \\
\hline
\end{tabular}

Fig. 2 a All-cause and $\mathbf{b}$ cardiovascular mortality for detemir and glargine. CPRD Clinical Practice Research Datalink, detemir insulin detemir, glargine insulin glargine, ONS Office for National Statistics 


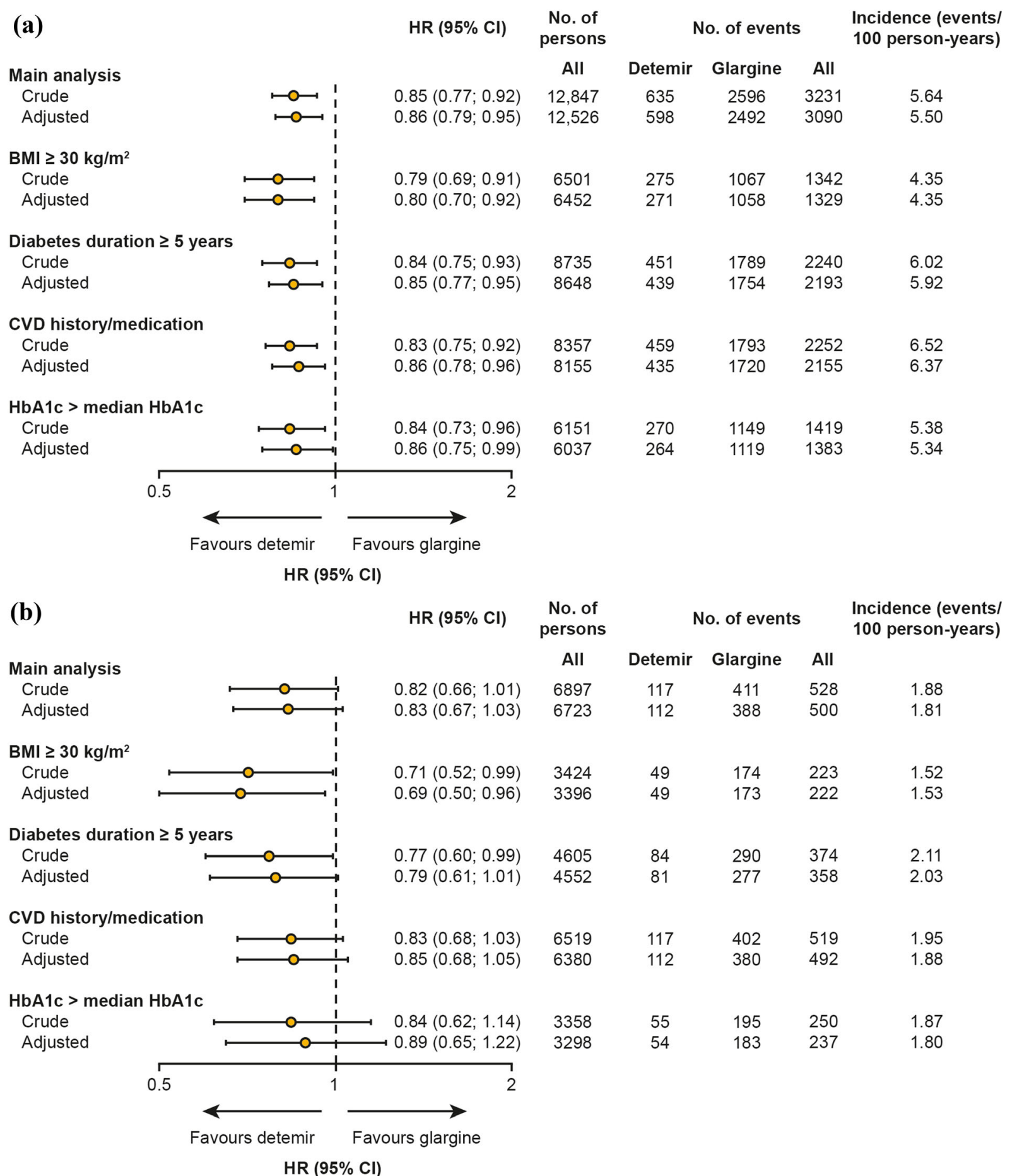

Fig. 3 Main analyses and sub-analyses for a all-cause and b cardiovascular mortality. Cox proportional hazards models were used to calculate crude and fully adjusted HRs (detemir/glargine), with age as the underlying timescale and the calendar year of the index date as a categorical variable. The (fully) adjusted models included a list of pre-specified covariates and relevant additional covariates that influenced the HR by $>10 \%$ on a log scale. The sub-analyses were undertaken in subsets of people with cardiovascular risk factors. $B M I$ body mass index, $C I$ confidence interval, detemir insulin detemir, glargine insulin glargine, $H b A l c$ glycated haemoglobin, $H R$ hazard ratio 
mortality, the crude and fully adjusted HRs $(95 \% \mathrm{CI})$ were $0.82(0.66 ; 1.01)$ and $0.83(0.67$; 1.03), respectively, with the non-significant numerical trends in favour of detemir (Fig. 3b).

\section{Sub-analyses}

Across all the subsets of people with cardiovascular risk factors, detemir was associated with a significantly lower risk of all-cause mortality when compared with glargine (Fig. 3a). Results of the sub-analyses for cardiovascular mortality tended to be consistent with the main analyses: a non-significant numerical trend towards a lower risk in people who initiated detemir than in those who initiated glargine (Fig. 3b). These associations were most pronounced in people with a $\mathrm{BMI} \geq 30 \mathrm{~kg} / \mathrm{m}^{2}$; for all-cause mortality, the crude and fully adjusted HRs (95\% CI) were $0.79(0.69 ; 0.91)$ and $0.80(0.70 ; 0.92)$, respectively. For cardiovascular mortality, the crude and fully adjusted HRs (95\% CI) were 0.71 (0.52; $0.99)$ and 0.69 (0.50; 0.96), respectively.

\section{Sensitivity Analyses}

Results of the sensitivity analyses are presented in Supplementary Fig. S2. The point estimates for the HRs comparing detemir with glargine for all-cause or cardiovascular mortality were similar across all of the sensitivity analyses. For allcause mortality, the significance of associations was unchanged across all the sensitivity analyses, with the exception of the adjusted 'current prescription' sensitivity analysis 4 (Supplementary Fig. S2).

\section{DISCUSSION}

In this UK-based real-world study, there was an association between all-cause mortality and basal insulin choice in insulin-naïve people with T2D; the mortality risk was significantly lower (by 14\%) with detemir versus glargine after adjustment for potential confounders. Additionally, there was a non-significant trend towards a $17 \%$ lower risk of cardiovascular mortality associated with detemir versus glargine, after adjustment for potential confounders. In general, these findings were consistent in subsets of people with cardiovascular risk factors and in sensitivity analyses that varied methodological assumptions. In sub-analyses, the associations between basal insulin choice and mortality risk were most pronounced in people with obesity; there were significant adjusted risk reductions of $20 \%$ and $31 \%$ for all-cause and cardiovascular mortality, respectively, with detemir compared with glargine.

A recent population-based study in Finland investigated the differences in all-cause and cause-specific mortality rates between new users of detemir and glargine who had T2D and were aged $\geq 40$ years [11]. The results from that study showed the HR (95\% CI) for all-cause and cardiovascular mortality for detemir versus glargine to be $0.71(0.54 ; 0.93)$ and $0.64(0.43$; $0.95)$, respectively [11]. These findings concur with, but are more pronounced than, those in the current study. This observation may be due to the Finnish study including only glargine U100, whereas users of both glargine U100 and U300 formulations were included in the current study. The follow-up time and covariates tested in the current study also differed from those in the Finnish study.

In sub-analyses, the associations between basal insulin choice and mortality risk were most pronounced in people with obesity (BMI $\geq 30 \mathrm{~kg} / \mathrm{m}^{2}$ ). One potential reason underlying associations between lower all-cause or cardiovascular mortality and the initiation of detemir rather than glargine in people with T2D and obesity is the lower weight gain that has been documented with detemir relative to glargine in people with diabetes $[8,9,18,19]$. Thus, people with diabetes and obesity may be at a comparatively lower risk of subsequent CVD when taking detemir (as compared with glargine). While the present analysis is unable to answer this question directly, the observation that the HR was numerically lowest in people with obesity is of interest and may warrant further investigation. A previous analysis of RCT data found that the magnitude of the difference in weight gain with detemir and other basal insulins increased with patients' BMI [20]. Thus, it is plausible that this effect might have contributed to the relative cardiovascular mortality benefit. 
Another potential contributing factor to the difference in HR is that the two basal insulins differ in their pharmacokinetic/pharmacodynamic profiles. Compared with neutral protoamine Hagedorn (NPH) insulin, which has highly variable absorption, tending to peak 4-5 $\mathrm{h}$ after injection, both detemir and glargine have a slower action, less pronounced peaks and a more consistent glucose-lowering effect over time [21, 22].

The present study has limitations, including that we were not able to completely account for confounding by indication. There may have been misclassification due to the assumption that "no information" in the register indicates "no exposure" for some of the variables, and this may not always have been true. The information in primary care records can be incomplete. The CPRD represents a UK real-world clinical practice population with respect to age, sex, BMI and ethnicity,; however, not necessarily with respect to geography and practice size [23]. Detemir may have been chosen (as per guidelines) for people who have concerns about hypoglycaemia or weight gain; therefore, the results may underestimate the benefits. This study included patients treated with either glargine U100 or U300 and it may not be appropriate to combine these products into a single treatment group as they differ at least in terms of their pharmacokinetic profiles [24]. We only had information available on prescriptions and did not know whether people collected the medication from the pharmacy and adhered to it.

This study also has strengths. For instance, the analyses were based on reliable data from a large national register that enabled assessment of a large cohort with a long duration of followup. The CPRD Gold database is the largest, most established and most utilised primary care database in the UK [17], and the information stored within the database has been extensively validated (reviewed in [25]). The data were adjusted for several potential confounding factors, and the sensitivity analyses, enabled by the large number of deaths, demonstrated that the HRs for mortality were robust to changes in the analysis methodology. As observational studies face methodological challenges, including confounding by indication, a new-user study design could help to mitigate these challenges by improving the validity of the study findings [26]. Furthermore, our findings are in line with those available from previous studies. Lastly, the total exposure time was relatively high.

\section{CONCLUSIONS}

Overall, the results from the present real-world study in the UK have shown that there was an association between all-cause mortality and basal insulin choice in insulin-naïve people with T2D; there was a significantly lower mortality risk with detemir versus glargine after adjustment for potential confounders. Additionally, there was a non-significant trend towards a lower risk of cardiovascular mortality with detemir versus glargine, again after adjustment for potential confounders. Of potential interest, the lower mortality risk associated with detemir was most pronounced in a subset of people with obesity.

\section{ACKNOWLEDGEMENTS}

This study is based in part on data from the CPRD obtained under license from the UK Medicines and Healthcare products Regulatory Agency. The data are provided by patients and collected by the National Health Service as part of their care and support. The interpretation and conclusions contained in this study are those of the authors alone. This study is based in part on ONS data contained within the CPRD database (via linkage) and we acknowledge ONS as the provider of the ONS data. Copyright (c) (2021), re-used with the permission of The Health and Social Care Information Centre. All rights reserved. The interpretation and conclusions contained in this study are those of the authors alone.

Funding. This study was supported by Novo Nordisk, which also funded this journal's Rapid Service Fee. 
Medical Writing and Editorial Assistance. The authors thank Sejal Varsani and Clare Lowe (Watermeadow Medical, an Ashfield Company, part of UDG Healthcare plc.) for providing medical writing and editorial support. This support was funded by Novo Nordisk.

Authorship. All authors confirm that they meet the International Committee of Medical Journal Editors (ICJME) uniform requirements for authorship and that they have contributed to: collection of data, critical analysis and interpretation of the data, drafting/critically revising the article and sharing in the final responsibility for the content of the manuscript and the decision to submit it for publication. LH is the guarantor of this work and, as such, had full access to all data in the study and takes responsibility for the integrity of the data.

Prior Presentation. Parts of this study were presented as an ePoster at the Diabetes UK Professional Conference, 18-20 March 2020, Glasgow, UK.

Disclosures. Lise Lotte N. Husemoen, Per K. Christensen and Niels V. Hartvig are employees and shareholders in Novo Nordisk A/S. At the time of the study, Lina S. Mørch was an employee of Novo Nordisk A/S but has since taken a position outside of Novo Nordisk A/S. Michael D. Feher reports advisory panels for Novo Nordisk, Sanofi and Eli Lilly; research support from Novo Nordisk and AstraZeneca; and speakers' bureaux for AstraZeneca, Novo Nordisk, Sanofi and Eli Lilly.

Compliance with Ethics Guidelines. This study's protocol was approved by the Independent Scientific Advisory Committee (ISAC) (Medicine and Healthcare Products Regulatory Agency): approved study protocol number 19_058A.

Data Availability. The datasets generated during and/or analysed during the current study are available from the corresponding author on reasonable request. The study protocol has been published. All data requests should specify how the data are planned to be used and will need to be approved by the authors and CPRD before data release.

Open Access. This article is licensed under a Creative Commons Attribution-NonCommercial 4.0 International License, which permits any non-commercial use, sharing, adaptation, distribution and reproduction in any medium or format, as long as you give appropriate credit to the original author(s) and the source, provide a link to the Creative Commons licence, and indicate if changes were made. The images or other third party material in this article are included in the article's Creative Commons licence, unless indicated otherwise in a credit line to the material. If material is not included in the article's Creative Commons licence and your intended use is not permitted by statutory regulation or exceeds the permitted use, you will need to obtain permission directly from the copyright holder. To view a copy of this licence, visit http://creativecommons.org/licenses/by$\mathrm{nc} / 4.0 /$.

\section{REFERENCES}

1. Fowler MJ. Microvascular and macrovascular complications of diabetes. Clin Diabetes. 2008;26: 77-82.

2. Trikkalinou A, Papazafiropoulou AK, Melidonis A. Type 2 diabetes and quality of life. World J Diabetes. 2017;8:120-9.

3. Davies MJ, D'Alessio DA, Fradkin J, Management of Hyperglycaemia in Type 2 Diabetes, et al. A consensus report by the American Diabetes Association (ADA) and the European Association for the Study of Diabetes (EASD). Diabetologia. 2018;2018(61): 2461-98.

4. Heise T, Pieber TR. Towards peakless, reproducible and long-acting insulins. An assessment of the basal analogues based on isoglycaemic clamp studies. Diabetes Obes Metab. 2007;9:648-59.

5. Becker RH, Dahmen R, Bergmann K, Lehmann A, Jax T, Heise T. New insulin glargine 300 Units · mL1 provides a more even activity profile and prolonged glycemic control at steady state compared with insulin glargine 100 Units . mL-1. Diabetes Care. 2015;38:637-43. 
6. Francesca Porcellati GBB, Carmine GF. Pharmacokinetics and pharmacodynamics of basal insulins. Diabetes Technol Ther. 2011;13:S15-24.

7. Poon K, King AB. Glargine and detemir: safety and efficacy profiles of the long-acting basal insulin analogs. Drug Healthc Patient Saf. 2010;2:213-23.

8. Rosenstock J, Davies M, Home PD, Larsen J, Koenen C, Schernthaner G. A randomised, 52-week, treatto-target trial comparing insulin detemir with insulin glargine when administered as add-on to glucose-lowering drugs in insulin-naive people with type 2 diabetes. Diabetologia. 2008;51:408-16.

9. Hollander P, Cooper J, Bregnhøj J, Pedersen CB. A 52-week, multinational, open-label, parallel-group, noninferiority, treat-to-target trial comparing insulin detemir with insulin glargine in a basal-bolus regimen with mealtime insulin aspart in patients with type 2 diabetes. Clin Ther. 2008;30:1976-87.

10. Meneghini LF, Rosenberg KH, Koenen C, Merilainen MJ, Lüddeke H-J. Insulin detemir improves glycaemic control with less hypoglycaemia and no weight gain in patients with type 2 diabetes who were insulin naive or treated with NPH or insulin glargine: clinical practice experience from a German subgroup of the PREDICTIVE study*. Diabetes Obes Metab. 2007;9:418-27.

11. Strandberg AY, Hoti FJ, Strandberg TE, Christopher S, Haukka J, Korhonen P. All-cause and cause-specific mortality among users of basal insulins NPH, detemir, and glargine. PLoS ONE. 2016;11:e0151910.

12. Fleming TR, DeMets DL. Surrogate end points in clinical trials: are we being misled? Ann Intern Med. 1996;125:605-13.

13. Grigore B, Ciani O, Dams F, et al. Surrogate Endpoints in Health Technology Assessment: an international review of methodological guidelines. Pharmacoeconomics. 2020;38:1055-70.

14. Cohen AT, Goto S, Schreiber K, Torp-Pedersen C. Why do we need observational studies of everyday patients in the real-life setting? Eur Heart J Suppl. 2015;17:D2-8.

15. Lawlor DA, Tilling K, Davey SG. Triangulation in aetiological epidemiology. Int $\mathrm{J}$ Epidemiol. 2016;45:1866-86.

16. CPRD. CPRD linked data. 2019. https://www.cprd. com/linked-data\#HES\%20Outpatient\%20data.

Accessed Dec 2019.
17. Williams T, van Staa T, Puri S, Eaton S. Recent advances in the utility and use of the General Practice Research Database as an example of a UK Primary Care Data resource. Ther Adv Drug Saf. 2012;3:89-99.

18. Raskin P, Gylvin T, Weng W, Chaykin L. Comparison of insulin detemir and insulin glargine using a basal-bolus regimen in a randomized, controlled clinical study in patients with type 2 diabetes. Diabetes Metab Res Rev. 2009;25:542-8.

19. Swinnen SG, Dain M-P, Aronson R, et al. A 24-week, randomized, treat-to-target trial comparing initiation of insulin glargine once-daily with insulin detemir twice-daily in patients with type 2 diabetes inadequately controlled on oral glucose-lowering drugs. Diabetes Care. 2010;33:1176-8.

20. Hollander PA. Insulin detemir for the treatment of obese patients with type 2 diabetes. Diabetes Metab Syndrome Obes Targets Ther. 2012;5:11-9.

21. Goldman-Levine JD, Lee KW. Insulin detemir-a new basal insulin analog. Ann Pharmacother. 2005;39:502-7.

22. Heinemann L, Linkeschova R, Rave K, Hompesch B, Sedlak M, Heise T. Time-action profile of the longacting insulin analog insulin glargine (HOE901) in comparison with those of NPH insulin and placebo. Diabetes Care. 2000;23:644-9.

23. Herrett E, Gallagher AM, Bhaskaran K, et al. Data resource profile: Clinical Practice Research Datalink (CPRD). Int J Epidemiol. 2015;44:827-36.

24. Becker RHA, Dahmen R, Bergmann K, Lehmann A, Jax T, Heise T. New insulin glargine 300 Units. $\mathrm{mL}-1$ provides a more even activity profile and prolonged glycemic control at steady state compared with insulin glargine 100 Units.mL-1. Diabetes Care. 2015;38:637-43.

25. Herrett E, Thomas SL, Schoonen WM, Smeeth L, Hall AJ. Validation and validity of diagnoses in the General Practice Research Database: a systematic review. Br J Clin Pharmacol. 2010;69:4-14.

26. Yoshida K, Solomon DH, Kim SC. Active-comparator design and new-user design in observational studies. Nat Rev Rheumatol. 2015;11:437-41. 